\title{
Intoxicación accidental por paraíso (Melia azedarach) en bovinos
}

\author{
Fazzio, L.E. ${ }^{1}$; Costa, E.F.'; Streitenberger, N. ${ }^{2}$; Pintos, M.E. ${ }^{1}$; Quiroga, M.A. ${ }^{2}$ \\ ${ }^{1}$ Hospital Escuela, ${ }^{2}$ Laboratorio de Patología Especial, Facultad de Ciencias Veterinarias, \\ Universidad Nacional de La Plata, calles 60 y 118, La Plata (1900), Provincia \\ Buenos Aires, Argentina. E-mail: fazzio@fcr.unlp.edu.ar
}

\begin{abstract}
Resumen
Fazzio, L.E.; Costa, E.F.; Streitenberger, N.; Pintos, M.E.; Quiroga, M.A.: Intoxicación accidental por paraíso (Melia azedarach) en bovinos. Rev. vet. 26: 1, 54-58, 2015. El presente trabajo describe un caso de intoxicación accidental de bovinos por Melia azederach en la Provincia de Buenos Aires, Argentina. Los signos clínicos observados fueron dificultad en la marcha, especialmente de miembros pelvianos, indiferencia al estímulo, decúbito permanente y muerte. El estudio histopatológico de las muestras de músculo esquelético obtenidas en la necropsia reveló diversos grados de degeneración y fragmentación segmentaria de las miofibras. Algunas de las fibras musculares evidenciaron pérdida de las estriaciones y tinción homogénea, débilmente eosinofílica, del sarcoplasma. En el intestino delgado se observó marcada necrosis linfocitaria en las placas de Peyer, siendo también evidente en linfocitos de la pulpa blanca del bazo, folículos linfoides del linfonódulo mesentérico y tejido linfoide asociado a los bronquios. Las enzimas musculares se encontraron muy por encima de sus valores normales. Sobre la base de los datos epidemiológicos, signos clínicos, hallazgos anatomopatológicos y resultados de análisis complementarios de laboratorio se arribó al diagnóstico de intoxicación por M. azedarach.
\end{abstract}

Palabras clave: bovino, intoxicación, paraíso, Melia azedarach.

\begin{abstract}
Fazzio, L.E.; Costa, E.F.; Streitenberger, N.; Pintos, M.E.; Quiroga, M.A.: Accidental intoxication with China berry (Melia azedarach) in bovine. Rev. vet. 26: 1, 54-58, 2015. This study describes a case of accidental poisoning with Melia azederach in cattle in Buenos Aires Province, Argentina. The clinical signs were staggering gait -mainly in the pelvic limbs- indifference to stimulus, permanent decubitus and death. The histopathological study of the skeletal muscle samples obtained from necropsy showed different degrees of segmental degeneration and fragmentation of muscle fibers. Some myofibers evidenced myofibrillar lisis and homogeneous, slightly eosinophilic, sarcoplasm stain. In the small intestine, severe lymphocyte necrosis in the Peyer's patches was observed. The latter was also noticeable in the splenic white pulp, in the lymphoid follicles of the mesenteric lymph node, and in the bronchial-associated lymphoid tissue. Muscle enzymes were higher than the reference values. Considering epidemiologic data, clinical signs, pathological findings and results from complementary laboratory analysis, $M$. azederach poisoning in cattle was diagnosed.
\end{abstract}

Key words: cattle, poisoning, China berry, Melia azedarach.

\section{INTRODUCCIÓN}

Melia azedarach (MA), también conocida como Paraíso, China berry tree, Ku-lian, Cinamomo o Persian lilac tree, es un árbol nativo del continente asiático con amplia distribución en el Norte de Australia, Estados Unidos, América del Sur y sur de Europa 1, 16,19. Posee hojas caducas que caen al principio del otoño y el

Programa de Incentivos UNLP 11/V222

Recibido: 10 septiembre 2014 - Aceptado: 3 noviembre 2014 fruto es una drupa de forma globosa, de $1,5 \mathrm{~cm}$ de diámetro, color verde, que toma un color amarillo pálido con el paso del tiempo ${ }^{8}$.

Desde la antigüedad, MA ha sido utilizada en la medicina tradicional china como agente antiparasitario y antifúngico de administración tópica y oral ${ }^{16}$. De igual modo, en la medicina ayurveda india, distintas partes de la planta (raíces, semillas, hojas, flores y frutos) son utilizadas para el tratamiento de resfríos, dolores de cabeza, desórdenes estomacales, procesos inflamatorios, diabetes, malaria y diversos cuadros de intoxicación ${ }^{3}$. 
En la actualidad, distintos trabajos dan cuenta de la acción antiparasitaria, antifúngica ${ }^{5,8,18}$, antiviral ${ }^{2}$, antioxidante ${ }^{15} \mathrm{y}$ antimicrobiana de MA, habiéndose identificado actividad contra organismos patógenos tales como Pseudomonas aeruginosa, Staphilococcus aureus, Escherichia coli y hongos como Aspergillus flavus, Aspergillus niger y Fusarium oxysporum ${ }^{17}$. Contrariamente a las propiedades preventivas y terapéuticas mencionadas, existen ocasionales descripciones de casos de intoxicación por MA en el hombre ${ }^{16}$.

En medicina veterinaria, MA es más conocida por su capacidad tóxica que por sus propiedades benéficas ${ }^{20}$. La toxicidad de la planta ha sido descripta en caninos, porcinos, bovinos, ovinos, cabras, conejos y pollos ${ }^{8,13}$. Los casos de intoxicación son consecuencia del consumo de hojas y/o frutos de MA ${ }^{8,12,13}$. La toxicidad depende de una combinación de 3 o 4 compuestos principales y otras 20 sustancias presentes en pequeñas cantidades. Los principales compuestos tóxicos son tetranortriterpenos de la clase limonoides citotóxicos, conocidos como meliatoxinas A1, A2, B1 y B2, las que se presentan mayormente concentrados en los frutos y actúan como enterotoxinas y neurotoxinas ${ }^{3,8,13}$. Si bien se cuenta con información referente a los efectos tóxicos de MA en distintas especies animales, se trata en mayor medida de la reproducción de cuadros de intoxicación experimental, no siendo numerosas las descripciones de casos de intoxicación natural 6,8,9,12,21 .

El presente trabajo describe un caso de intoxicación aguda en bovinos por ingestión accidental de frutos de MA.

\section{MATERIAL Y MÉTODOS}

En el mes de julio de 2013 se recibió una consulta por un caso de mortandad de terneros en un establecimiento dedicado a la cría y recría bovina ubicado en el Partido de General Las Heras, Provincia de Buenos Aires, Argentina. El lote estaba conformado por 156 terneros, raza Aberdeen Angus y Aberdeen Angus x Hereford, de 6 a 8 meses de edad y un peso promedio aproximado de $160 \mathrm{Kg}$.

Los animales se encontraban en un potrero con buena disponibilidad forrajera a base de pasto natural, en un sistema extensivo. El día 4 de julio el personal del establecimiento identificó un animal muerto, observando además dos animales en decúbito permanente y cinco animales con marcada dificultad en la marcha, más notoria en los miembros pelvianos. Frente a esta situación se convocó a un profesional veterinario quien realizó la necropsia de un animal e informó el hallazgo de frutos de MA en el contenido ruminal y la ausencia de lesiones anatomopatológicas relevantes.

El día 5 de julio se realizó la exploración clínica de los animales afectados y se procedió a colectar muestras de sangre y orina. En la anamnesis se informó que, en el potrero en el que estaban alojados los animales, se había realizado la poda en días previos de una hilera de árboles de MA ubicados en proximidad al alambrado perimetral, arrojándose las ramas cortadas dentro del potrero.

Al momento de la visita se identificaron 7 animales con signos clínicos y 2 animales muertos. Además del examen clínico se obtuvieron muestras de sangre en tubos con EDTA y sin anticoagulante, por punción de la vena yugular de tres animales que presentaban signos, para realizar estudios hematológicos y enzimáticos. Además se recolectó orina, por micción espontánea, de uno de los animales afectados, realizándose el análisis físico químico y estudio del sedimento urinario, dentro de las 6 h de extracción.

Se realizó la necropsia completa de un animal y se recolectaron muestras para estudio histopatológico de diferentes órganos: rumen, librillo, red, bazo, linfonódulo mesentérico, intestino delgado, pulmón, corazón, riñón e hígado así como de músculos de distintas regiones (serrato, tríceps, bíceps femoral, recto interno, psoas, semimembranoso, semitendinoso, dorsal ancho y diafragma). Las muestras se fijaron en formol neutro al $10 \%$ y se tiñeron con hematoxilina y eosina, examinándose por microscopía óptica.

\section{RESULTADOS Y DISCUSIÓN}

$\mathrm{Al}$ recorrer el potrero, se constató la presencia de abundante cantidad de ramas con frutos de MA, algunas presentando rastros de consumo. Además de los signos relatados por el propietario se observó apatía, tremores musculares, incoordinación en la marcha -especialmente de miembros pelvianos-, depresión, falta de respuesta al estímulo y micciones frecuentes. Uno de los animales presentó pleurostótono. La motilidad ruminal, así como la temperatura corporal se ubicaron dentro del rango normal, pero las frecuencias cardíaca y respiratoria se encontraron aumentadas.

A la necropsia se observó abundante espuma en la luz de la tráquea y múltiples hemorragias epicárdicas y endocárdicas. En el contenido ruminal se constató la presencia de gran cantidad de drupas (frutos) de MA (Figura 1). La mucosa del rumen, retículo, omaso, abomaso e intestino delgado presentó color rojo oscuro en toda la extensión. Los músculos de los miembros torá-

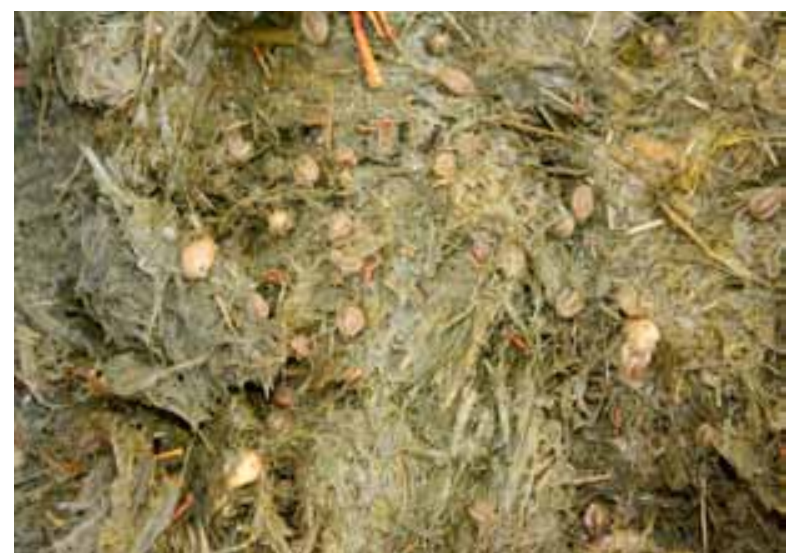

Figura 1. Contenido ruminal. Se observa la presencia de gran cantidad de drupas (frutos) de Melia azedarach. 


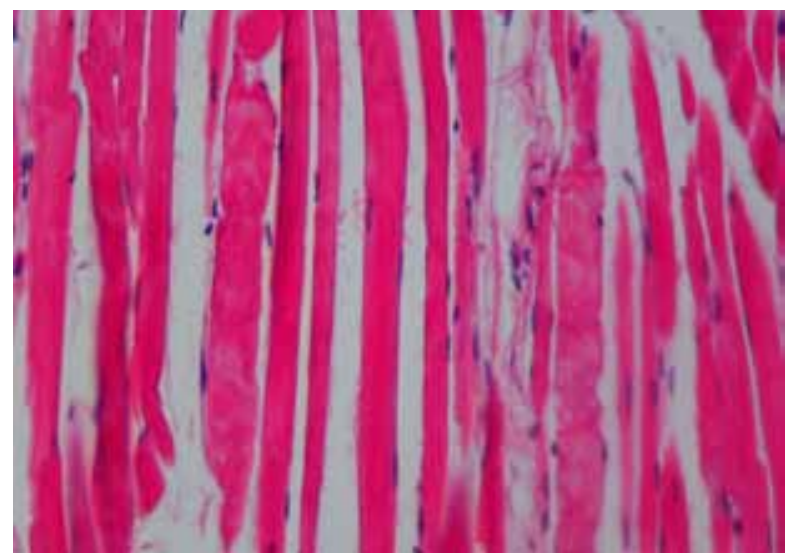

Figura 2. Músculo esquelético. Fibras musculares con sarcoplasma homogéneo, débilmente eosinofílico, ausencia de miofibrillas y núcleo (degeneración hialina incipiente). H\&E 40x.

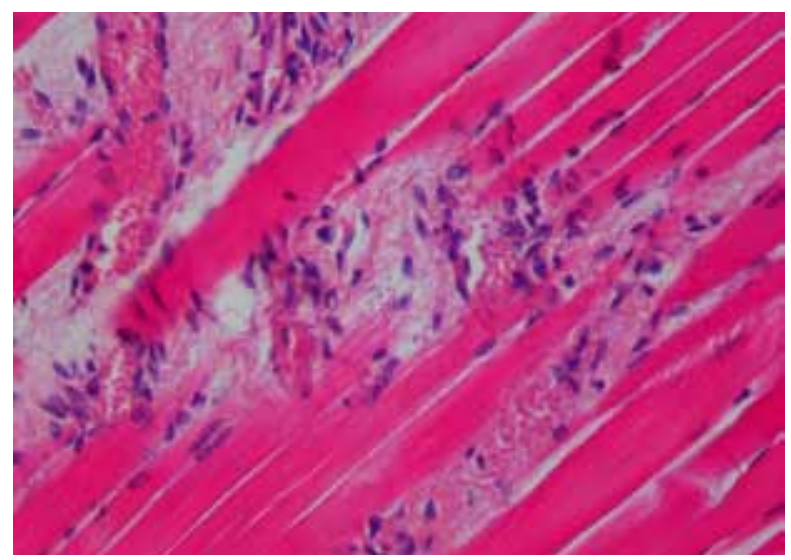

Figura 3. Músculo esquelético. Foco de fragmentación de miofibras con escaso infiltrado de macrófagos. H\&E 40x.

Tabla 1. Recuento de leucocitos y fórmula leucocitaria absoluta (miles $/ \mathrm{mm}^{3}$ ) en terneros intoxicados.

\begin{tabular}{lcccc}
\hline recuento & caso 1 & caso 2 & caso 3 & ref. \\
\hline leucocitos tot. & 22.000 & 28.800 & 33.400 & $4.000-12.000$ \\
neutrófilos cay. & 1.760 & 2.880 & 2.004 & $>120$ \\
neutrófilos segm. & 17.380 & 22.752 & 27.388 & $600-4.000$ \\
eosinófilos & 0 & 0 & 0 & $0-2.400$ \\
basófilos & 0 & 0 & 0 & $0-2$ \\
linfocitos & 2.420 & 2.304 & 3.006 & $2.500-7.500$ \\
monocitos & 440 & 864 & 1002 & $25-850$ \\
\hline
\end{tabular}

ref: valor de referencia, tot.: totales, cay.: en cayado, segm.: segmentados.

Tabla 2. Actividades enzimáticas en suero de animales $(\mathrm{n}=3)$ con signos clínicos de toxicidad (valores en $\mathrm{UI} / 1)$.

\begin{tabular}{lcccc}
\hline enzima & caso 1 & caso 2 & caso 3 & ref. \\
\hline CPK & 1300 & 765 & 16 & $\leq 228$ \\
AST & 988 & 605 & 29 & $78-132$ \\
GGT & 400 & 807 & 22 & $5-48$ \\
\hline
\end{tabular}

CPK: creatinfosfoquinasa, AST: aspartatoamino transferasa, GGT: gammaglutamil transferasa, ref: valor de referencia. cico y pelviano se observaron de color rosado pálido a blanquecino, más manifiesto en los músculos semitendinoso y semimembranoso.

El examen histopatológico reveló cambios en los distintos músculos esqueléticos de los que se obtuvieron muestras. Las lesiones presentaron distribución multifocal consistiendo en pequeñas áreas con distinto grado de degeneración de las miofibras. Se observaron pequeñas zonas de fragmentación y miolisis, evidenciándose, en algunos segmentos, pérdida de las estriaciones y tinción homogénea, débilmente eosinofílica del sarcoplasma y, en ocasiones, escaso infiltrado de macrófagos (Figuras 2 y 3 ).

El corazón presentó hemorragias subepicárdicas y el pulmón, extensas áreas de congestión y edema. En los pre-estómagos e intestino la mucosa evidenció una leve a moderada congestión con conservación del epitelio. En el intestino delgado se observó, además, marcada necrosis/apoptosis linfocitaria en las placas de Peyer (Figura 4), siendo también evidente en la pulpa blanca del bazo, folículos linfoides del linfonódulo mesentérico y, en menor medida, en el tejido linfoide asociado a los bronquios (BALT) intrapulmonares. El riñón presentó abundantes cilindros hialinos en la luz tubular y escasos túbulos contorneados con necrosis parcial o total del epitelio.

Los resultados del estudio hematológico evidenciaron una marcada leucocitosis con neutrofilia y desviación izquierda regenerativa, así como ligera monocitosis (Tabla 1). El resto de los parámetros hematológicos no presentaron alteraciones, encontrándose dentro del rango de referencia para la especie ${ }^{10}$. Las variaciones de las actividades enzimáticas de creatinfosfoquinasa (CPK), aspartato aminotransferasa (AST), y gammaglutamil transpeptidasa (GGT) se exponen en la Tabla 2. El análisis de orina reveló densidad de 1018, pH 8 , alta concentración de proteínas $(300 \mathrm{mg} / \mathrm{dl})$ y en el sedimento urinario se encontró abundante cantidad de células epiteliales de transición.

El diagnóstico de intoxicación por plantas se basa en los antecedentes del caso, los signos clínicos predominantes, los hallazgos de necropsia, la evidencia del consumo del vegetal involucrado, la presencia del mismo en el contenido gastrointestinal y -cuando el principio tóxico es conocido- su determinación por técnicas analíticas ${ }^{4}$.

En el presente caso el diagnóstico de intoxicación por MA se realizó, sin mayores dificultades, sobre la base de los datos epidemiológicos, signos clínicos, hallazgos anatomopatológicos macro y microscópicos, valores elevados de enzimas musculares en suero y presencia de abundante cantidad de drupas de MA en el contenido ruminal. Los hallazgos clínicos coinciden en su totalidad con los observados en la reproducción experimental de la intoxicación con frutos en bovinos ${ }^{13}$. La única excepción fue la ausencia de atonía ruminal, mencionada como un hallazgo constante.

Los hallazgos anatomopatológicos aquí descriptos concuerdan con los observados en los casos de repro- 


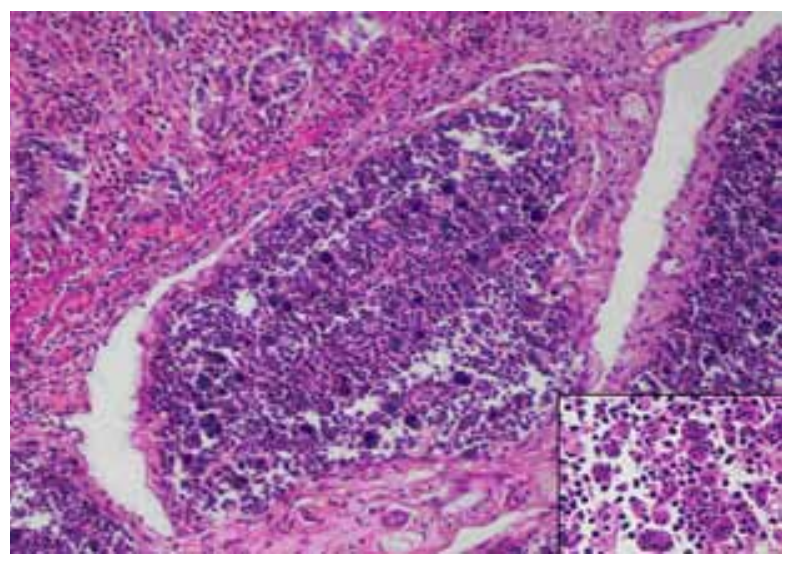

Figura 4. Intestino delgado. Marcada necrosis de linfocitos en placas de Peyer (H\&E 20x). Recuadro: detalle de macrófagos con cuerpos apoptóticos y detritus celulares intracitoplasmáticos. H\&E 100x.

ducción experimental de la intoxicación en bovinos y porcinos con hojas y frutos ${ }^{12,13}$. Entre las lesiones microscópicas resultó evidente la necrosis/apoptosis de los linfocitos tanto en los órganos linfoides como en el tejido linfoide asociado a mucosas (intestinal y bronquial).

Se ha descripto que algunos de los limonoides de MA poseen un potente efecto citotóxico en la línea celular HL60 (human promyelocytic leukemia cells) provocando apoptosis celular tanto por la vía mitocondrial como por la vía mediada por el receptor de muerte celular ${ }^{1}$. Esto explicaría el efecto radiomimético que MA provoca sobre los órganos linfoides. En cambio, y a diferencia de lo descripto por algunos autores ${ }^{12,13}$, no se observó necrosis del epitelio de los órganos del tubo digestivo ni lesiones degenerativas hepáticas, sino sólo cambios congestivos en los pre-estómagos e intestino.

La presencia de abundantes cilindros hialinos y ocasional necrosis en los túbulos renales coincide con la detección de proteínas en orina, sin embargo no se conoce de qué modo los principios tóxicos de MA puedan afectar la función renal. Las lesiones de los músculos esqueléticos concuerdan con anteriores descripciones de intoxicaciones experimentales en bovinos, tanto con hojas como con frutos de este árbol ${ }^{12,13}$. La presentación multifocal y monofásica de las lesiones resulta esperable en un cuadro tóxico agudo ${ }^{11} \mathrm{y}$ aunque no fueron muy extensas, coincidieron con un alto nivel de CPK y AST en suero.

CPK es una enzima específica del músculo estriado esquelético y cardíaco, que escapa de la célula muscular en procesos de degeneración o necrosis. El aumento sérico de la actividad enzimática puede ser considerado como un fidedigno indicio de lesión muscular ${ }^{14}$. AST también se localiza en el tejido muscular; sin embargo también se encuentra en otros tejidos, especialmente el hepático, por lo cual su incremento no necesariamente debe relacionarse con daño muscular. En tal sentido, la determinación de GGT, de localización hepática exclusiva, es útil para detectar injuria hepática ${ }^{14}$. En el presente caso el aumento de CPK y AST confirman la lesión muscular ya que los valores de GGT se encontraron dentro del rango considerado normal en concordancia con la ausencia de lesión hepática al estudio histopatológico.

La leucocitosis neutrofílica con desvío a la izquierda regenerativo es una respuesta del organismo frente a un proceso inflamatorio ${ }^{7}$. Este hallazgo, si bien estuvo presente en los tres animales estudiados, no se encuentra descrito en intoxicaciones experimentales por MA en la bibliografía consultada. Cabe destacar que este tipo de respuesta ocurre en animales no solo cuando se produce una respuesta frente a un agente infeccioso sino también en procesos inflamatorios tóxicos, como es el caso aquí presentado ${ }^{10}$.

\section{REFERENCIAS}

1. Akihisa T, Pan X, Nakamura Y, Kikuchi T, Takahashi N, Matsumoto M, Ogihara E, Fukatsu M, Koike K, Tokuda H. 2013 Limonoids from the fruits of Melia azedarach and their cytotoxic activities. Phytochem 89: 59-70.

2. Alche LE, Assad FK, Meo M, Coto CE, Maier MS. 2003. An antiviral meliacarpin from leaves of Melia azedarach. Naturforsch 58, 215-219.

3. Azam MM, Mamun AN, Towfique NM, Sen MK, Nasrin S. 2013 Pharmacological potentials of Melia azedarach L. Am J BioScience 1: 44-49.

4. Botha CJ, Penrith ML. 2008 Poisonous plants of veterinary and human importance in southern Africa. J Ethnopharmacol 119: 549-558.

5. Carpinella MA, Giorda LM, Ferrayoli CG, Palacios SM. 2003. Antifungal effects of different organic extracts from Melia azedarach $L$. on phytopathogenic fungi and their isolated active components. J Agric Food Chem 51: 2506-2511.

6. Cooper RG. 2007. Poisoning in ostriches following ingestion of toxic plants-field observations. Trop Anim Health Prod 6: 439-442.

7. Feldman BF, Zinkel JG, Jain NC. 2000. Schalm's Veterinary Hematology, 5th ed, Lippincott Williams and Wilkins, Canada, $1344 \mathrm{p}$.

8. Ferreiro D, Orozco JP, Mirón C, Real T, Hernández D, Soler F, Pérez M. 2010. Chinaberry tree (Melia azedarach) poisoning in dog: a case report. Top Companion Anim Med 25: 64-67.

9. Hare WR, Schutzman H, Lee BR, Knight MW. 1997. Chinaberry poisoning in two dogs. $J$ Am Vet Med Assoc 210: 1638-1640.

10. Kaneko JJ, Harvey W, Bruss ML. 1997. Clinical Biochemistry of Domestic Animals, 5th ed, Academic Press, San Diego, p. 890-894.

11. Van Vleet JF, Valentine BA. 2007. Muscle and Tendon In: Jubb, Kennedy and Palmer's Pathology of Domestic Animals (Maxie MG ed.), 5th ed., Saunders Elsevier, Philadelphia, p. 187-229.

12. Méndez MC, Aragao M, Elias F, Riet-Correa F, Gimeno EJ. 2002. Experimental intoxication by the leaves of Melia azedarach (Meliaceae) in cattle. Pesq Vet Bras 22: 19-24. 
13. Méndez MC, Elias F, Aragao M, Gimeno EJ, Riet-Correa F. 2002. Intoxication of cattle by the fruits of Melia azedarach. Vet Hum Toxicol 44: 145-148.

14. Meyer DJ, Harvey JW. 2007. Medicina Laboratorial Veterinaria, Ed. Multimédica, Barcelona, $452 \mathrm{p}$.

15. Mohd Fazil A, Mohd Ashwaq A, Hameed T, Khaja Z, Mohd I. 2008. Antioxidative activity of Melia azedarach leaf extract. Iranian J Pharm Therap 7: 31-34.

16. Phua DH, Tsai WJ, Ger J, Deng JF, Yang CC. 2008. Human Melia azedarach poisoning. ClinToxicol 46: 10671070.

17. Sen A, Batra A. 2012. Evaluation of antimicrobial activity of different solvent extracts of medicinal plant: Melia azedarach. Int J Curr Pharm R 4: 67-73.
18. Szewczuk VD, Mongelli ER, Pomilio AB. 2003. Antiparasitic activity of Melia azedarach growing in Argentina. Mol Med Chem 1: 54-57.

19. Tokarnia CH, Brito MF, Barbosa JD, Peixoto PV, Döbereiner J. 2012. Plantas Tóxicas do Brasil para Animais de Produção, Ed. Helianthus, Río de Janeiro, 586 p.

20. Wynn SG, Fougere BJ. 2007. Veterinary Herbal Medicine, Ed. Mosby Elsevier, Philadelphia, 536 p.

21. Zakir-ur-Rahman, Ahmad S, Qureshi S, Atiq-urRahman, Badar Y. 1991. Toxicological studies of Melia azedarach L. (flowers and berries). Pak J Pharm Sci. 4: 153-158.

\section{Revista Veterinaria obtuvo el máximo nivel de categorización del CAICYT-CONICET}

Tras el pertinente proceso de evaluación según criterios de calidad editorial, en setiembre de 2005 CAICYT-CONICET ha clasificado a nuestra publicación con Categoría 1 (nivel superior de excelencia), con lo cual pasa a integrar el Catálogo Latindex (folio 14022). La Dirección de Revista veterinaria agradece a quienes colaboraron para obtener tan importante distinción. Ver: http://www.latindex. unam.mx/busquedas/catalogotitulo.html 